\title{
Part A: The Development of mI SMART, a Nurse-Led Technology Intervention for Multiple Chronic Conditions
}

\author{
Jennifer A. Mallow ${ }^{1}$, Laurie A. Theeke ${ }^{1}$, Rebecca Walls ${ }^{2}$, Elliott Theeke ${ }^{1}$, Brian K. Mallow ${ }^{3}$ \\ ${ }^{1}$ School of Nursing, West Virginia University, Morgantown, West Virginia, USA \\ ${ }^{2}$ Milan Puskar Health Right, Morgantown, West Virginia, USA \\ ${ }^{3}$ Sovern Run, LLC, Albright, West Virginia, USA \\ Email: jamallow@hsc.wvu.edu, Itheeke@hsc.wvu.edu, etheeke@hsc.wvu.edu, bkmallow@gmail.com
}

Received 29 March 2016; accepted 25 April 2016; published 28 April 2016

Copyright (C) 2016 by authors and Scientific Research Publishing Inc.

This work is licensed under the Creative Commons Attribution International License (CC BY). http://creativecommons.org/licenses/by/4.0/

(c) (i) Open Access

\section{Abstract}

Background: The treatment of Multiple Chronic Conditions (MCC) is complex for both patients and providers. Used as integrated tools, technology may decrease complexity, remove the barrier of distance to obtain care, and improve outcomes of care. A new platform that integrates multiple technologies for primary health care called mI SMART (Mobile Improvement of Self-Management Ability through Rural Technology) has been developed. The purpose of this paper is to present to development of mI SMART, a nurse-led technology intervention for treating for MCC in primary care. Methods: The creation of mI SMART was guided by the model for developing complex nursing interventions. The model suggests a process for building and informing interventions with the intention of effectiveness, sustainability, and scalability. Each step in the model builds from and informs the previous step. Results: The process resulted in the integrated technologies of $\mathrm{mI}$ SMART. The system combines a HIPAA compliant, web-based, structure of mHealth sensors and mobile devices to treat and monitor multiple chronic conditions within an existing free primary care clinic. The mI SMART system allows patients to track diagnoses, medications, lab results, receive reminders for self-management, perform self-monitoring, obtain feedback in real time, engage in education, and attend visits through video conferencing. The system displays a record database to patients and providers that will be integrated into existing Electronic Health Records. Conclusion: By using the model for developing complex nursing interventions, a multifaceted solution to clinical problems was identified. Through modeling and seeking expert review, we have established a sustainable and scalable integrated nurse-led intervention that may increase access and improve outcomes for patients living in rural and underserved areas. The first trial of $\mathrm{mI}$ SMART has been completed and evaluated for feasibility, acceptability, and effectiveness in persons in rural areas living with multiple chronic conditions.

"Corresponding author. 
Keywords

Multiple Chronic Conditions, mHealth, Telehealth, Health Disparities, Nursing Informatics

\section{Introduction}

People living with chronic conditions suffer from poor health, disability, and pre-mature death [1]. It is estimated that 117 million (half) of US adults have a chronic condition, and 1 in 4 adults have Multiple Chronic Conditions (MCC) [2]. In addition, most health-care expenditures in the United States are due to chronic conditions [3]. Due to the complexity of treatments, conflicting advice for each condition, and co-existing lack of the social, health and behavioral determinants of health due to disparities, individuals with MCC often have difficulty in achieving treatment goals [4]-[7]. Therefore, simplifying treatment regimens, managing conflicting advice, and assessing determinants of heath such as addressing the availability of resources, access to healthcare, and improved diet physical activity are imperative to improving outcomes and self-management ability.

Healthcare technology is developing rapidly and may offer an opportunity to enhance care. Use of technology can improve healthcare systems' ability to monitor patients without travel, coordinate the services of multiple healthcare providers, and manage patient symptoms in real time [5] [8]. In addition, technology interventions augment care and management of MCC by improving access to care and improving patient outcomes. The subsequent long-term effects of technology use lead to diminished health disparities and reduced healthcare costs [9]. However, much of the available healthcare technology intended to treat MCCs is developed by private industry and used in non-academic settings. In the current literature, widespread reporting of development process is not present. Therefore, questions remain about acceptability, feasibility and efficacy across populations.

Some examples of existing patient facing technology interventions include: Access to information from electronic medical records, requesting medication refills and appointments through automated systems, communicating with healthcare providers using secure messing systems, managing specific chronic conditions using telehealth, using personal health records to track progress, and interacting with on-line support groups using social media [10]. The available literature on these individual technology interventions is promising and does provide limited evidence of improving outcomes, cost effectiveness and cultural relevance [11]. However, the lack of integration of data into existing systems and healthcare records, lack of reimbursement for technology services, and the necessity to access multiple technology interventions individually, increase complexity for both healthcare systems and patients and decrease widespread use for patients with MCC.

Nurses are uniquely trained to address the complexity of caring for the whole person as an individual in the context of their family, community, and environment. The Institute of Medicine (IOM) calls nurses to practice to the full extent of their education and to be partners in redesigning heath care in the United States [12]. As patient advocates who store, maintain and communicate data and information [13], nurses are trained to use validated processes to develop complex interventions, to think about patients within systems, and to implement and improve interventions [14]. The purpose of this paper is to present the development of mI SMART (Mobile Improvement of Self-Management Ability through Rural Technology), a nurse-led technology intervention for treating for MCC in primary care.

\section{Methods for Intervention Development}

The model for developing complex nursing interventions [15] guided the overall process of intervention development. The model was developed and refined from the Medical Research Council (MRC) frame-work for developing complex interventions [16] and other guidelines that contribute additional guidance to inform the development of nursing interventions. The model suggests a process for building and informing interventions with the intention of effectiveness, sustainability, and scalability. Each step in the model builds from and informs the previous step. The steps include: problem identification, practice analysis, identification of the overall objective, identification of theory or key principles, building and planning, modelling and seeking expert review, and developing the study protocol.

\subsection{Problem Identification \& Practice Analysis}

The first phase of mI SMART development, problem identification, was undertaken through a needs, practice 
and policy analysis. The identified problem originally started with a concern for the poor outcomes of persons with diabetes in a rural clinic serving uninsured and underinsured individuals. A retrospective review of the combined pharmacy records, front desk scheduling system, and Electronic Health Record (EHR) found that diabetes patients who lived further than 30 miles from the clinic and had more than one chronic condition missed appointments compared to those who live closer and had only one chronic condition [17]. Based on these findings, the research team decided that an intervention was needed that addressed MCCs and overcame the disparity of access to care due to distance. A search of the empirical evidence related to eliminating the burden of distance revealed the use of mHealth tools as a potential intervention to improve care [10]. In addition, it was also noted that policies for federal payers were improving and Medicare pays for services that provide live, interactive videoconferencing [18]. Hence, the decision was made to begin to develop an intervention that incorporated mHealth tools with live videoconferencing.

\subsection{Identification of the Overall Objective}

The overall objective of the mI SMART project is to improve quality of life and outcomes of care for rural and underserved individuals living with multiple chronic conditions. This objective was determined using a series of activities. First, the thoughts and ideas of nurses, including in-patient, out-patient, advanced practice, and researchers were sought. In addition, conversations intentionally included physicians, pharmacists, social workers, health educators, computer scientists, engineers, and private industry leaders. This objective will be accomplished by improving access, self-management ability, and communication with use of technology within a healthcare system that is trusted by patients.

\subsection{Identification of the Theory or Key Principles}

A substantive review of potential foundational theories was conducted. Two models came forward as having relevance and utility in the development of mI SMART. The first model, the Quality Health Outcomes Model, was selected for its broad concepts that could be conceptualized and adapted based on the system, patient population, and evolving intervention [19]. The second model chosen, the Chronic Care Model, assists in understanding how to build an intervention that changes healthcare delivery at the healthcare system level [20].

The Quality Health Outcomes Model was developed by the American Academy of Nursing's Expert Panel on Quality Health Care in 1996 as an expansion of Donabedian's structure-process-outcome framework. The four major concepts are: system, interventions, patients, and outcomes. The model is a dynamic framework that recognizes the reciprocal relationship that occurs between patients, the system where care is provided, and interventions [21]. Outcomes are linked to the interactions of a patient with the healthcare system and with healthcare interventions that are focused on the individual, family, or community [19]. Interventions are affected by both system and patient characteristics in producing desired outcomes.

The Chronic Care Model consists of six interrelated system changes meant to make patient-centered, evidence based care easier to accomplish [22]. The major concepts in the model are: health system, community support, self-management support, decision support, clinical information systems, and delivery system design [23]. This model is operationalized through a prepared healthcare team delivering planned interactions, self-management support with effective use of community resources, integrated decision support, and supportive information technology (IT) which are designed to work together to strengthen the provider-patient relationship, improve communication and improve health outcomes [23]. Therefore, the development of mI SMART is based on the major concepts and underlying assumptions of the Quality Health Outcomes Model and the Chronic Care Model.

\subsection{Building, Planning \& Modeling}

Based on the guiding frameworks, problem identification and overall goals of the intervention, the first version of the mI SMART platform was modeled and a basic plan for implementation was developed. The expertise of an information technology specialist was sought to complete the programming involved. While the initial intervention was planned to be implemented in a free clinic in a rural location, the thought of expanding the intervention was a consideration. The decision was made to make the platform web-based as opposed to a system specific application (app). Hence, the first iteration of mI SMART was web-based, HIPAA compliant, system that 
includes the use of self-monitoring devices, video conferencing capabilities, real-time feedback, automatic patient specific reminders for self-monitoring and medication, links and video education for chronic illness, and secure messaging.

\subsection{Seeking Expert Review}

After a model of the mI SMART platform was developed, expert review was sought by holding focus groups for in various settings [11]. The participants invited to the focus groups were intended to represent both the first users of the system and future potential users. The mI SMART platform was demonstrated to each group and specific questions were used to elicit conversation and feedback. After participation in the focus groups, participants were asked to complete surveys. Focus groups were attended by 37 individuals and surveys were completed by 29 healthcare team members including 7 males and 22 females, age range 23 to 62. The participants included: Medical Assistants, Registered Nurses, front desk staff, Physicians, Nurse Practitioners, Physician's Assistants, Pharmacists, Social Workers, Administrators, and Board Members. The focus group participants identified perceived obstacles of patient use as: ability, willingness, and time. System obstacles were identified as: lack of integration, lack of reimbursement, and cost. Many positive attributes of mI SMART were identified and included: capability for virtual visits, readability, connectivity, user-friendliness, ability to capture biophysical measures, enhanced patient access, and incorporation of multiple technologies. Providers suggested increasing capability for biophysical and symptom monitoring for more common chronic conditions.

\subsection{Developing the Study Protocol}

Based on the feedback provided in the focus groups, changes were made to the mI SMART system prior to developing the study protocol. The system combines a HIPAA compliant, web-based, structure of mHealth sensors and mobile devices to treat and monitor multiple chronic conditions. The mI SMART system allows patients to track diagnoses, medications, lab results, receive reminders for self-management, perform self-monitoring, obtain feedback in real time, engage in education, and attend visits through video conferencing. The system displays a record database to patients and providers that will be integrated into existing Electronic Health Records. Once these changes were made, the study protocol was developed and funding for the project was sought and obtained. The first trial of the mI SMART platform has been completed. The model for developing complex nursing interventions will be used to guide the refinement of the mI SMART platform prior to a larger randomized trial.

\section{Discussion}

The use of the model for developing complex nursing interventions was essential to the successful development of a robust, adaptive and empirically ground technology platform (see Figure 1 for the operationalization of the Model for Developing Complex Interventions in Nursing). The mI SMART platform is an improvement over the currently available systems. Combining multiple health sensors, education, reminders, video conferencing, lab results, and secure messaging removes the necessity to access multiple technology interventions individually. This combination of services decreases complexity of care for both healthcare systems and patients. The intervention has been implemented with 30 adult participants living in a rural area who have MCCs and are experiencing difficulties attending clinic visits. Feasibility and acceptability for both the patients and healthcare providers was evaluated and reported in Part B. In addition, efficacy of the intervention was evaluated with patient outcomes which will be reported in a separate paper. The first implementation of mI SMART was targeted to a specific population and clinic. Future use of the mI SMART platform should be adapted to other populations and practice settings. The content of the platform in its present iteration is reflective of current empirical evidence about the use of technology, a specific needs and practice analysis, and has been adapted based on feedback of a wide, but not exhaustive, variety of healthcare providers.

\section{Conclusion}

Our long-term objective is to create a new and substantive departure from the status quo by integrating multiple mHealth tools into one platform within an existing rural health clinic to go beyond traditional office visits and shifting to real-time exchanges between patients and providers across geographical boundaries. An efficacious 


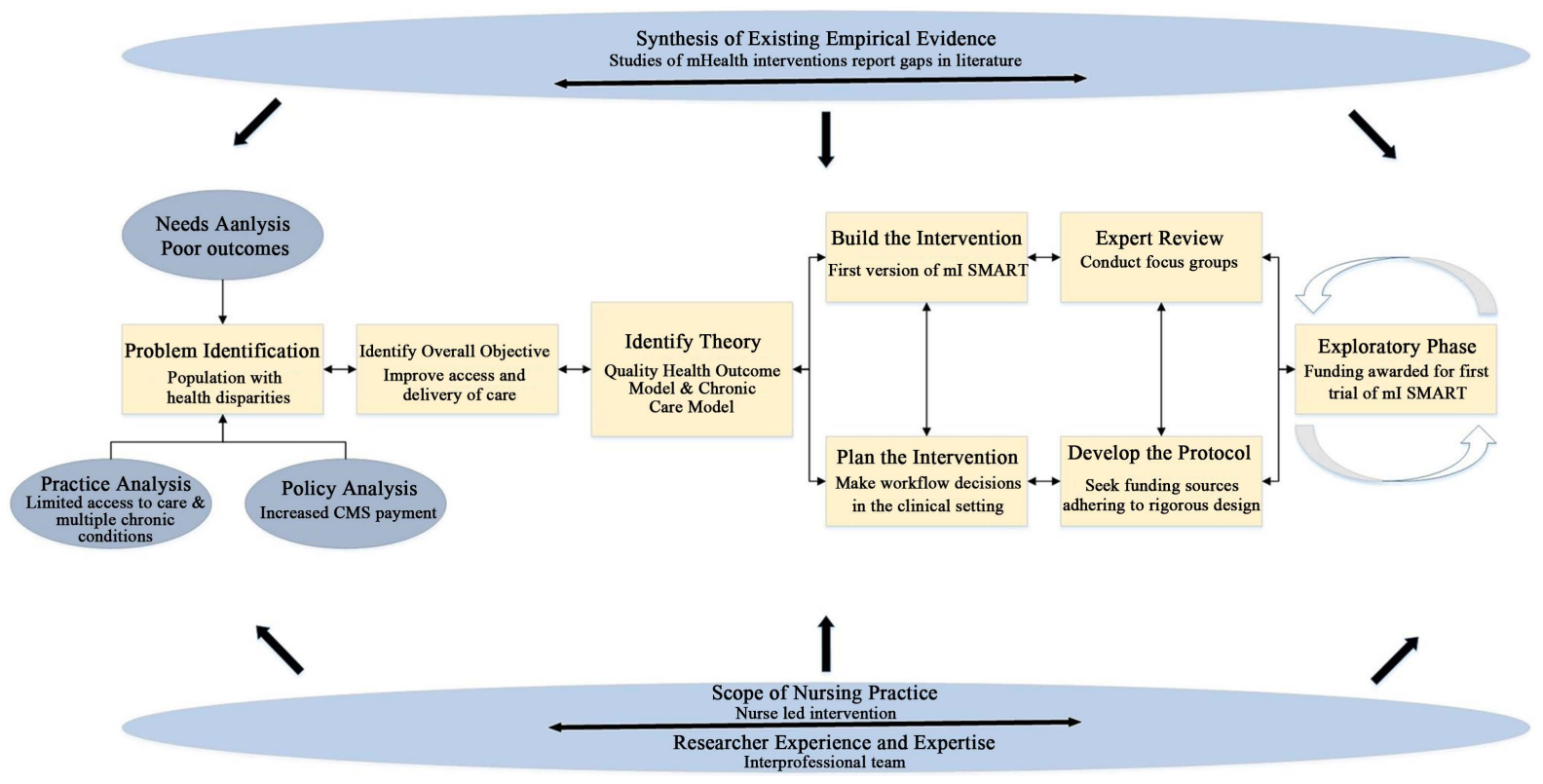

Figure 1. Operationalization of the model for developing complex interventions in nursing. This figure is based on the model found in [15].

shift in the traditional rural healthcare delivery paradigm to one that uses technology is expected to result. The model for developing complex nursing interventions is currently being used to update mI SMART based on patient and provider feedback and integration into the EHR is planned. The initial feasibility and acceptability of the mI SMART platform is published in Part B of this article. Other plans include fully disseminating the results of the first implementation of mI SMART, pursuing commercialization and seeking funding for the larger trial.

\section{Acknowledgements}

This project was supported by the Robert Wood Johnson Foundation Nurse Faculty Scholars Program. The Robert Wood Johnson Foundation Nurse Faculty Scholars Program is working to develop the next generation of national leaders in academic nursing through career development awards for outstanding junior nursing faculty. The program aims to strengthen the academic productivity and overall excellence of nursing schools by providing mentorship, leadership training and salary and research support to faculty.

\section{References}

[1] Centers for Disease Contro and Prevention. Death and Mortality. National Center for Health Statistics 2013.

[2] Ward, B.W., Schiller, J.S. and Goodman, R.A. (2014) Peer Reviewed: Multiple Chronic Conditions Among US Adults: A 2012 Update. Preventing Chronic Disease, 11, E62.

[3] Bauer, U.E., Briss, P.A., Goodman, R.A. and Bowman, B.A. (2014) Prevention of Chronic Disease in the 21st Century: Elimination of the Leading Preventable Causes of Premature Death and Disability in the USA. The Lancet, 384, 45-52. http://dx.doi.org/10.1016/S0140-6736(14)60648-6

[4] Grembowski, D., Schaefer, J., Johnson, K.E., Fischer, H., Moore, S.L., Tai-Seale, M., et al. (2014) A Conceptual Model of the Role of Complexity in the Care of Patients with Multiple Chronic Conditions. Medical Care, 52, S7-S14. http://dx.doi.org/10.1097/MLR.0000000000000045

[5] LeRoy, L., Bayliss, E., Domino, M., Miller, B.F., Rust, G., Gerteis, J., et al. (2014) The Agency for Healthcare Research and Quality Multiple Chronic Conditions Research Network: Overview of Research Contributions and Future Priorities. Medical Care, 52, S15-S22. http://dx.doi.org/10.1097/MLR.0000000000000095

[6] Magnan, E.M., Palta, M., Mahoney, J.E., Pandhi, N., Bolt, D.M., Fink, J., et al. (2015) The Relationship of Individual Comorbid Chronic Conditions to Diabetes Care Quality. BMJ Open Diabetes Research \& Care, 3, e000080. http://dx.doi.org/10.1136/bmjdrc-2015-000080

[7] Tinetti, M.E. and Basu, J. (2014) Research on Multiple Chronic Conditions: Where We Are and Where We Need to 
Go. Medical Care, 52, S3-S6. http://dx.doi.org/10.1097/MLR.0000000000000093

[8] Khairat, S., Wijesinghe, N., Wolfson, J., Scott, R. and Simkus, R. (2014) Building a Multicenter Telehealth Network to Advance Chronic Disease Management. In: Mantas, J., Househ, M.S. and Hasman, A., Eds., Integrating Information Technology and Management for Quality of Care, Vol. 202, IOS Press, Amsterdam, 299-302.

[9] Shi, L., Chen, C.-C., Nie, X., Zhu, J. and Hu, R. (2014) Racial and Socioeconomic Disparities in Access to Primary Care among People with Chronic Conditions. The Journal of the American Board of Family Medicine, 27, 189-198. http://dx.doi.org/10.3122/jabfm.2014.02.130246

[10] Mallow, J.A., Theeke, L.A., Barnes, E.R., Whetsel, T. and Mallow, B.K. (2014) Using mHealth Tools to Improve Rural Diabetes Care Guided by the Chronic Care Model. Online Journal of Rural Nursing and Health Care, 14, 43. http://dx.doi.org/10.14574/ojrnhc.v14i1.276

[11] Mallow, J.A., Theeke, L.A., Long, D.M., Whetsel, T., Theeke, E. and Mallow, B.K. (2015) Study Protocol: Mobile Improvement of Self-Management Ability through Rural Technology (mI SMART). SpringerPlus, 4, 423. http://dx.doi.org/10.1186/s40064-015-1209-y

[12] Institute of Medicine (2011) Committee on the Robert Wood Johnson Foundation Initiative of the Future of Nursing. The Future of Nursing: Leading Change, Advancing Health.

[13] Dykes, P.C., Hurley, A., Cashen, M., Bakken, S. and Duffy, M.E. (2007) Development and Psychometric Evaluation of the Impact of Health Information Technology (I-HIT) Scale. Journal of the American Medical Informatics Association, 14, 507-514. http://dx.doi.org/10.1197/jamia.M2367

[14] Hoyt, R.E. and Yoshihashi, A.K. (2014) Health Informatics: Practical Guide for Healthcare and Information Technology Professionals. Lulu.com, Raleigh, 27.

[15] Corry, M., Clarke, M., While, A.E. and Lalor, J. (2013) Developing Complex Interventions for Nursing: A Critical Review of Key Guidelines. Journal of Clinical Nursing, 22, 2366-2386. http://dx.doi.org/10.1111/jocn.12173

[16] Craig, P., Dieppe, P., Macintyre, S., Michie, S., Nazareth, I. and Petticrew, M. (2008) Developing and Evaluating Complex Interventions: The New Medical Research Council Guidance. British Medical Journal, 337, a1655.

[17] Mallow, J.A., Theeke, L.A., Barnes, E.R., Whetsel, T. and Mallow, B.K. (2014) Free Care Is Not Enough: Barriers to Attending Free Clinic Visits in a Sample of Uninsured Individuals with Diabetes. Open Journal of Nursing, 4, 912. http://dx.doi.org/10.4236/ojn.2014.413097

[18] Gilman, M. and Stensland, J. (2013) Telehealth and Medicare: Payment Policy, Current Use, and Prospects for Growth. Medicare \& Medicaid Research Review, 3, No. 4. http://dx.doi.org/10.5600/mmrr.003.04.a04

[19] Mitchell, P.H., Heinrich, J., Moritz, P. and Hinshaw, A.S. (1997) Outcome Measures and Care Delivery Systems. Introduction and Purposes of Conference. Medical Care, 35, NS1-NS5. .

[20] Wagner, E.H. (1998) Chronic Disease Management: What Will It Take To Improve Care for Chronic Illness? Effective Clinical Practice, 1, 2-4.

[21] Mitchell, P.H., Ferketich, S. and Jennings, B.M. (1998) Quality Health Outcomes Model. American Academy of Nursing Expert Panel on Quality Health Care. Image_-The Journal of Nursing Scholarship, 30, 43-46. http://dx.doi.org/10.1111/j.1547-5069.1998.tb01234.x

[22] Roger, V.L., Go, A.S., Lloyd-Jones, D.M., Benjamin, E.J., Berry, J.D., Borden, W.B., et al. (2012) Heart Disease and Stroke Statistics - 2012 Update: A Report from the American Heart Association. Circulation, 125, e2-e220. http://dx.doi.org/10.1161/CIR.0b013e31823ac046

[23] Pullicino, P.M., McClure, L.A., Howard, V.J., Wadley, V.G., Safford, M.M., Meschia, J.F., et al. (2011) Identifying a High Stroke Risk Subgroup in Individuals with Heart Failure. Journal of Stroke and Cerebrovascular Diseases, 22, 620-626. 